\title{
688 KNOCKOUT OF THE INHIBITORY RECEPTOR TIGIT ENHANCES ANTI-TUMOR RESPONSE OF EX VIVO EXPANDED NK CELLS
}

Tayler Croom-Perez*, Md Faqrul Hasan, Thomas Dieffenthaller, Liza Robles-Carrillo, Jonathan Eloriaga, Alicja Copik. University of Central Florida, Orlando, FL, United States

Background Natural Killer (NK) cells are an important immune cell population crucial for the success of many immunotherapies due to their critical role in both the innate response and in priming an adaptive immune response. Recently, much focus has been on generating highly cytotoxic NK cells for use in adoptive cell therapy and combinatorial immune-oncology therapies. The robust cytotoxicity against cancer cells and NK cell activation relies on fine tuning of activating and inhibitory signals. NK cell inhibitory receptors are often upregulated upon stimulation and activation and can be a marker for exhaustion. One of the major NK inhibitory receptors, T-cell immunoglobulin and ITIM domain (TIGIT), is highly expressed in ex vivo expanded NK cells. In this study, we will investigate if knockout of TIGIT in ex vivo expanded NK cells will enhance their anti-tumor activity.

Methods CRISPR was used to make a targeted TIGIT knockout (KO) in ex vivo expanded NK cells. TIGIT KO NK cells were then compared to wild type NK cells to determine any changes in phenotypic markers. IFN $\gamma$, TNF $\alpha$, and the degranulation marker CD107a expression were analyzed after coculture with cancer cells. Cytotoxicity of TIGIT KO NK cells was compared to wild type NK cells against multiple different cancer cell spheroids using a kinetic live-cell imaging assay. Multiple NK cell:target cell ratios were analyzed over time to determine killing half-time and maximum killing. Data were fit to dose-response curves to determine cytotoxicity EC50 values.

Results CRISPR was used to efficiently knockout TIGIT in ex vivo expanded NK cells and decreased expression levels to less than 5\%. After co-culture with Raji cells expressing the TIGIT ligand PVR (CD155), TIGIT KO NK cells showed increased expression of IFN $\gamma, \mathrm{TNF} \alpha$ and CD107a. TIGIT KO NK cells showed improved killing compared to wild type NK cells. TIGIT KO cells killed more target cells faster with significant decreases in half-killing time and more than a 2-fold decrease in EC50 cytotoxicity values in $3 \mathrm{D}$ spheroid cytotoxicity models against six different cancer cell lines. When NK cell:target cell ratios were low, the maximum cytotoxicity was also significantly higher in TIGIT KO cells.

Conclusions Knockout of the TIGIT gene in ex vivo expanded NK cells resulted in higher functioning NK cells with increased cytokine expression, degranulation, and cytotoxicity against multiple cancer cell lines. These TIGIT knockout NK cells with improved antitumor activity provide a promising universal effector population with the potential for enhanced therapeutic efficacy.

Acknowledgements We thank FL DOH Grant \#9JK04 for funding and MaxCyte for providing instrument for testing.

http://dx.doi.org/10.1136/jitc-2021-SITC2021.688 